\title{
Let-7 miRNAs sensitize breast cancer stem cells to radiation-induced repression through inhibition of the cyclin D1/Akt1/Wnt1 signaling pathway
}

\author{
HUIFANG SUN, CHANGMAO DING, HUIYU ZHANG and JIANBO GAO \\ Department of Radiology, The First Affiliated Hospital of Zhengzhou University, Zhengzhou, \\ Henan 450052, P.R. China
}

Received April 18, 2015; Accepted July 12, 2016

DOI: $10.3892 / \mathrm{mmr} .2016 .5656$

\begin{abstract}
The tumor-suppressive let-7 family of microRNAs (miRNAs) has been previously identified to induce cell apoptosis, proliferation-inhibition and suppression of the self-renewal capacities of cancer stem cells (CSCs). However, let-7-mediated sensitization of tumors to radiation treatment remains to be investigated fully in triple negative breast cancer (TNBC), of which the clinical treatment is challenging. The inhibitory effect of let-7 miRNAs on the self-renewal ability of CSCs from TNBC was investigated. It was identified that radiation inhibited the self-renewal ability of TNBC stem cells by inhibiting cyclin D1 and protein kinase B (Akt1) phosphorylation. Let-7d stimulates radiation-induced tumor repression, exerting synergistic effects with radiotherapy on stem cell renewal. Through western blotting, immunofluorescence and a luciferase assay, it was identified that reduced cyclin D1/Akt1/wingless type MMTV integration site family member 1 (Wnt1) signaling activity accounts for the let-7-induced radiation sensitization. Let-7 directly inhibits cyclin D1 expression, resulting in low phosphorylation of Akt1, which is critical for the let-7-induced inhibition of mammosphere numbers. The let-7d-induced Akt1 inhibition contributed to tumor repression, with similar results to those obtained with Akt inhibitors. Furthermore, it was identified that the inhibition of Wnt1 is critical for the functioning of let-7d, and that addition of recombinant Wnt 1 abolished the effects of let-7d on sensitization to radiotherapy. Let-7d is suggested to be a promising therapeutic agent in the treatment of TNBC by targeting CSCs and sensitizing tumors to radiotherapy via inhibition of cyclin D1/Akt1/Wnt1 signaling.
\end{abstract}

Correspondence to: Professor Jianbo Gao, Department of Radiology, The First Affiliated Hospital of Zhengzhou University, 1 Jianshe East Road, Erqi, Zhengzhou, Henan 450052, P.R. China E-mail: cjr.gaojianbo@vip.163.com

Key words: let-7d, triple negative breast cancer, radiotherapy, cancer stem cells, Akt1, Wnt1

\section{Introduction}

Breast cancer is the most common malignancy observed amongst women, accounting for nearly a quarter of all cases, and outcomes vary depending on cancer type, extent of disease the and patient's age (1). Four main subtypes of breast tumor have been identified based on patterns of gene expression, including luminal $\mathrm{A} / \mathrm{B}$, human epidermal growth factor receptor 2 (HER2)+ [estrogen receptor (ER)-, progesterone receptor (PR)-] and triple negative tumors (ER-, PR- and HER-), which influence the therapeutic strategies used and the clinical prognosis (2). Endocrine therapies have been identified to be effective in treating breast cancer of luminal A and luminal B subtypes, and when used together with chemotherapy, the prognosis of patients was observed to be improved $(3,4)$. Breast cancer in young women is more likely to be an aggressive triple negative or HER2+ sub type, and is more likely to be identified at an advanced stage, due to the aggressive subtype (5). Trastuzumab has been demonstrated to improve prognosis of patients with overexpressed HER-2; however, effective treatment strategies for triple negative breast cancer (TNBC), which is resistant to common chemotherapies and does not respond well to endocrine therapies, remain to be identified. Radiotherapy is used as a remedial strategy, therefore increasing the sensitization of TNBC cells to radiation may improve the clinical prognosis and quality of life of patients (6).

MicroRNAs (miRNAs) are deregulated in the majority of malignancies, serving roles in tumor development and progression, and numerous miRNAs have been reported to influence the therapeutic response of cancer to clinical treatments $(7,8)$. In breast cancer, miR-17/20, miR-190, miR-200, miR-34, the let-7 family and additional miRNAs have been identified to be involved in the pathogenesis of tumor biology (7-9). The let-7 family was the earliest identified family of non-translational RNAs, of which the functions remain to be fully elucidated. Let-7 miRNAs act as suppressive genes through directly binding to the 3 'untranslated region (UTR) of Ras, interleukin-6, cyclin D1, mitogen-activated protein kinase, LIN28-A/B, c-Myc, DICER1 and numerous other oncogenes in ER-positive breast cancer $(7,10)$. However, the role of let-7 in TNBC, and the let-7-induced sensitization of tumor repression 
remains to be fully elucidated, which is important for the development of future clinical treatments. Let-7 miRNAs have been reported to limit the numbers of stem cells in normal and cancerous tissue samples, aiding in the maintenance of the differentiation of stem cells and cancer stem cells (CSCs), thus inhibiting tumor progression. Previous studies have identified that let-7 is able to stimulate chemotherapeutic effects, however, the effects of radiation combined with let-7 remain unclear, which require elucidation for the improvement in TNBC treatment $(11,12)$. The current study investigated the roles of let-7 and radiation on the stem cells of TNBC, and then assessed the combined effects of let-7d and radiation. The possible mechanisms associated with these effects were investigated, with the aim of improving treatment strategies for TNBC by overcoming recurrence via inhibition of the renewal ability of CSCs.

\section{Materials and methods}

Cell culture, transfection and infection. The human breast cancer cell lines ZR75-1, MCF-7, BT-20, HS587-T and MDA-MB-231 (MM-231) were purchased from the American Type Culture Collection (Manassas, VA, USA) and were cultured in Roswell Park Memorial Institute-1640 medium (Gibco; Thermo Fisher Scientific China, Beijing, China), containing $10 \%$ fetal bovine serum (Thermo Fisher Scientific China) and $1 \%$ penicillin and streptomycin (Gibco; Thermo Fisher Scientific China). The mammospheres were cultured in Dulbecco's modified Eagle's medium/Ham's F-12 medium (Corning Incorporated, Corning, NY, USA) supplemented with $10 \mathrm{ng} / \mathrm{ml}$ human basic fibroblast growth factor (Sigma-Aldrich, St. Louis, MO, USA), $10 \mathrm{ng} / \mathrm{ml}$ epidermal growth factor (Sigma-Aldrich), $1 \mu \mathrm{g} / \mathrm{ml}$ hydrocortisone (Sigma-Aldrich), $4 \mu \mathrm{g} / \mathrm{ml}$ insulin and $1 \%$ penicillin and streptomycin (13). All cells were cultured in $5 \% \mathrm{CO}_{2}$ at $37^{\circ} \mathrm{C}$. Oligonucleotides encoding let-7d mature miRNA and scramble control were synthesized and cloned into the pGLVU6/RFP vector (Shanghai GenePharma Co., Ltd., Shanghai, China). Transfections for the luciferase assay were performed using Lipofectamine 2000 reagent (Invitrogen; Thermo Fisher Scientific, Inc.) according to the manufacturer's instructions.

Reverse transcription-quantitative polymerase chain reaction $(R T-q P C R)$ and western blotting. Total RNA was isolated using TRIzol reagent (Invitrogen; Thermo Fisher Scientific, Inc.). cDNA (1 g) was prepared using a ReverTra Ace qPCR RT kit from Toyobo Co., Ltd. (Osaka, Japan). RT-qPCR was performed with the Thunderbird SYBR qPCR Mix (Toyobo Co., Ltd.) and a LightCycler 480 PCR system (Roche Diagnostics, Basel, Switzerland). The thermocycling conditions were as follows: Two repeats of $95^{\circ} \mathrm{C}$ for $30 \mathrm{sec}$; and 45 cycles of $95^{\circ} \mathrm{C}$ for $5 \mathrm{sec}$ and $60^{\circ} \mathrm{C}$ for $30 \mathrm{sec}$. Mature let-7d was quantified using a predesigned miRCURY LNA ${ }^{\mathrm{TM}}$ assay (Exiqon, Inc., Woburn, MA, USA) with the primer, 5'-AGAGGTAGTAGGTTGCATAGTT-3'. Expression of the U6 small nuclear RNA endogenous control was assayed for normalization. Gene expression determinations were made using the comparative $2^{-\Delta \Delta \mathrm{Cq}}$ method (14). The proteins were harvested in radioimmunoprecipitation assay lysis buffer (Beijing Biotech Co., Ltd., Beijing, China) and then subjected to $10 \%$ sodium dodecyl sulfate-polyacrylamide gel (Sigma-Aldrich) electrophoresis separation. The rabbit monoclonal antibody against Wnt1 (1:1,000; cat. no. 2530) was purchased from Cell Signaling Technology (Shanghai, China), monoclonal cyclin D1 (1:1,000; sc-753) and phosphorylated-protein kinase B (Akt1) (Ser473; 1:800; sc-7985-R) were purchased from Santa Cruz Biotechnology, Inc. (Shanghai, China). Antibodies against viniculin (1:3,000; ab18058) were obtained from Abcam (Cambridge, MA, USA).

Sphere formation assays. Cells of different groups were plated in ultra-low attachment (non-adherent condition) 6-well plates (Corning Incorporated) to test their ability of forming primary mammospheres. The mammosphere numbers were counted on day 10 and the mammosphere formation efficiency (MFE) was calculated as the percentage ratio between obtained spheres and plated cells $(10,15-17)$. Obtained mammospheres from the different groups were disaggregated then re-suspended to test their ability of self-renewal in the next generation. Akt1 inhibitors (Akti-1/2; ab142088; Abcam) and recombinant Wnt1 protein (50 ng/ml; Sigma-Aldrich) were used to identify the respective roles of Akt1 and Wnt1.

Immunofluorescence and luciferase assay. The cells were added into the chambers and were fixed with $10 \%$ formalin (Sigma-Aldrich). The cells were blocked with $2 \%$ normal goat serum (ab7481; Abcam), then were incubated with primary antibodies as above for $1 \mathrm{~h}$ at $4^{\circ} \mathrm{C}$ in phosphate-buffered saline (PBS; Sigma-Aldrich), then with a secondary antibody for $30 \mathrm{~min}$ at room temperature with Alexa Fluor ${ }^{\circledR} 488$ (goat anti-mouse IgG; 1:200; Abcam; cat. no. ab150113) or Alexa Fluor ${ }^{\circledR} 633$ (goat anti-rabbit IgG; 1:1,000; Invitrogen; Thermo Fisher Scientific, Inc.; cat. no. A-21071) at room temperature. Cells were then washed in PBS, incubated for 10 min with PureBlu $^{\mathrm{TM}}$ 4',6-Diamidino-2-Phenylindole Nuclear Staining Dye [135-1303; Bio-Rad Laboratories (Shanghai) Co., Ltd., China] and washed in PBS again. The luciferase reporter 3'UTR of cyclin D1, the T-cell factor (TCF) luciferase reporter and its mutant control, the promoter of $\beta$-catenin and the mutant control were designed and cloned into miRGLO by Promega Corporation (Madison, WI, USA). Cells were seeded at $30 \%$ confluence in 4 -well plates prior to transient transfection with FuGENE 6 reagent (Roche Diagnostics) (18).

Binding site predication. TargetScan (www.targetscan.org), was used to determine the mRNA target genes. If no target gene was identified, the existing binding site between the selected miRNA and target gene may be frail.

Statistical analysis. All data were obtained from three independent experiments, and are expressed as the mean \pm standard deviation. Statistical analysis was conducted with Student's t-test and the $\chi^{2}$ test using SPSS software, version 16.0 for Windows (SPSS, Inc., Chicago, IL, USA) and Excel 2007 (Microsoft Corporation, Redmond, WA, USA). $\mathrm{P}<0.05$ was considered to indicate a statistically significant difference. 
A

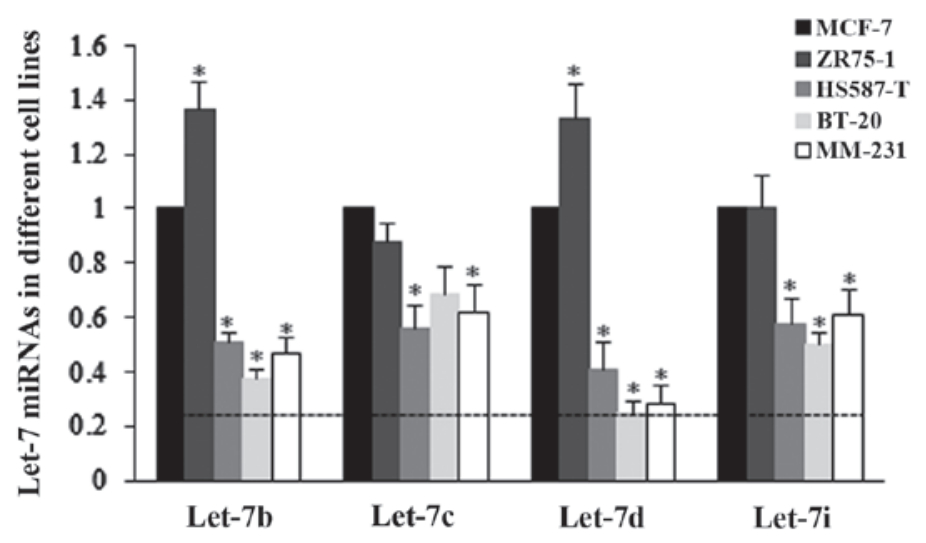

C

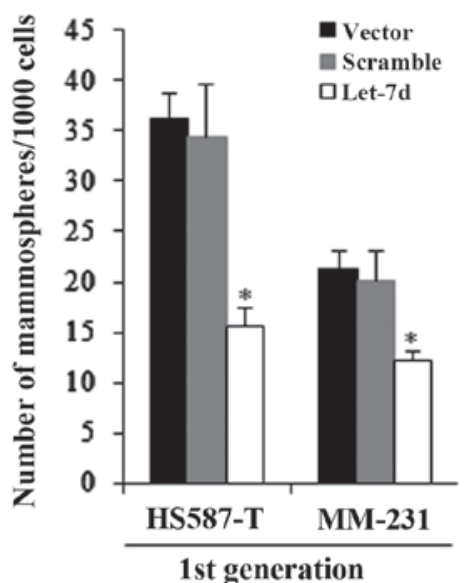

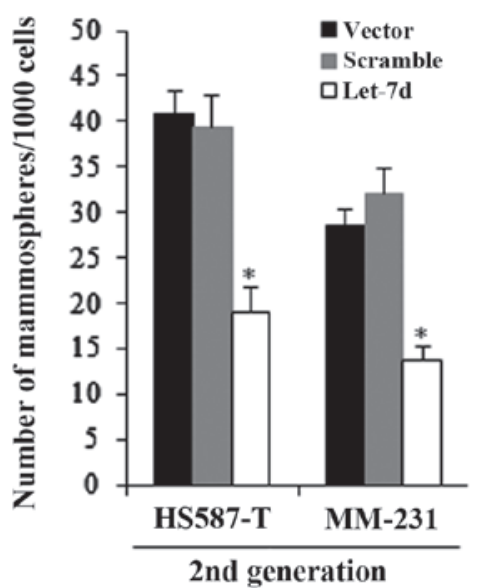

B
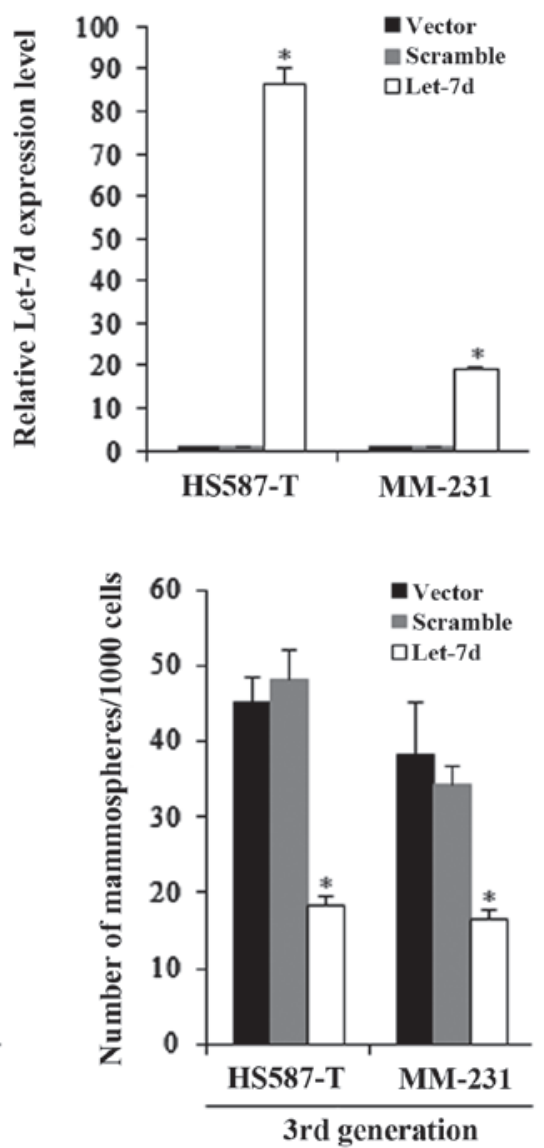

D

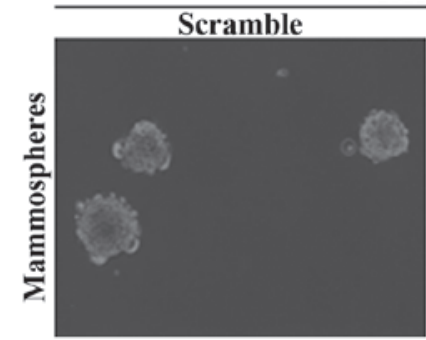

HS587-T
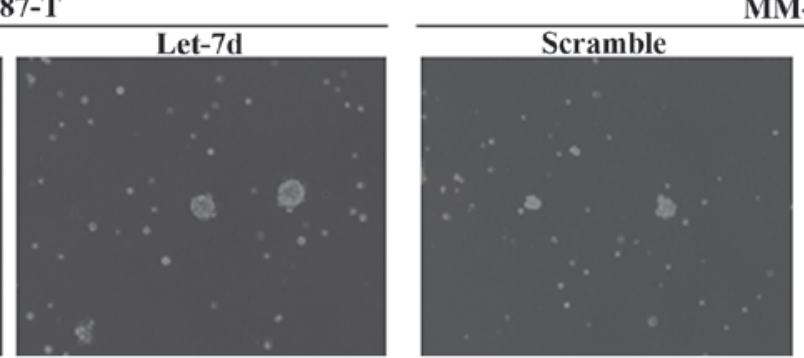

MM-231

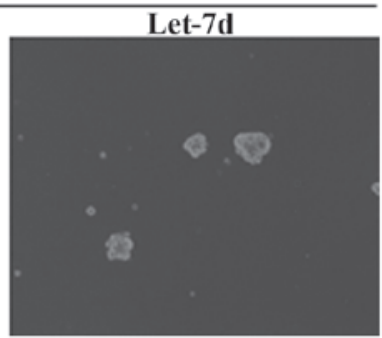

Figure 1. Let-7 miRNA signatures in triple negative breast cancer cells. The expression levels of let-7 miRNAs in multiple breast cancer cell lines were detected and calculated using the $2^{-\Delta \Delta C t}$ method, with U6 as the internal reference gene. (A) Let-7d was significantly reduced in the HS587-T and MM-231 triple negative breast cancer cell lines, compared with other cell lines. " $\mathrm{P}<0.01$ vs. the MCF-7 cell line. (B) Let-7d was effectively overexpressed in HS587-T and MDA-MB-231 cells. "P<0.01 vs. the scramble control. (C) Let-7d continuously significantly inhibited mammosphere formation in three generations. "P<0.01 vs. the scramble control. (D) Representative images of mammospheres acquired from two cell lines of triple negative breast cancer. miRNA, microRNA.

\section{Results}

The functions of let-7 miRNAs in TNBC cells. The let-7b, let-7c, let-7d and let-7i expression levels were assessed in multiple breast cancer cell lines, which had been previously reported to be significantly deregulated in breast cancer (19-21). The results indicated that let-7d was significantly reduced in TNBC in HS587-T and MM-231 cells, compared with the other cell lines $(\mathrm{P}<0.01$; Fig. 1A). The HS587-T and MM-231 cells were then infected with lentiviral-based let-7d vectors. The RFP-based let-7d lentiviral vector was successfully infected into HS587-T and MDA-MB-231 cells, and the expression levels were detected and presented in Fig. 1B. The effects of let-7d on the self-renewal ability of TNBC cells were evaluated by the mammosphere formation assay, and let-7d was identified to be able to significantly inhibit the mammosphere numbers, exerting a continuous repression of the self-renewal ability (Fig. 1C and D).

Let-7d sensitized TNBC stem cells to radiation-induced self-renewal repression. Different doses of radiotherapy and treating durations were assessed, and the results indicated that radiation could inhibit the self-renewal ability of TNBC stem cells (Fig. 2A-C). However, it was observed that 2 Gy radiation for $12 \mathrm{~h}$ did not significantly affect the MFE of HS587-T and MM-231 cells (Fig. 2A). In combination with let-7d, 2 Gy radiation exhibited a markedly stronger inhibition of the self-renewal ability of the two cell types, which was also significantly stronger than that of let-7d alone (Fig. 2D). Furthermore, the combined use of let- $7 \mathrm{~d}$ and 2 Gy radiation 
A

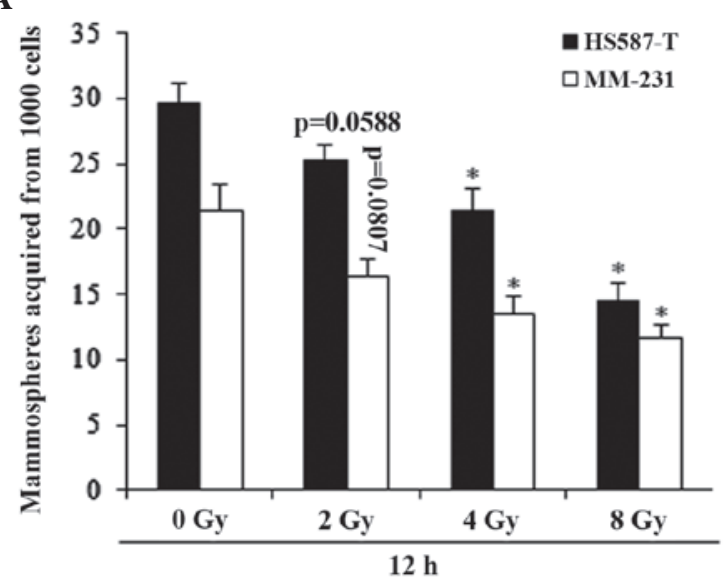

C

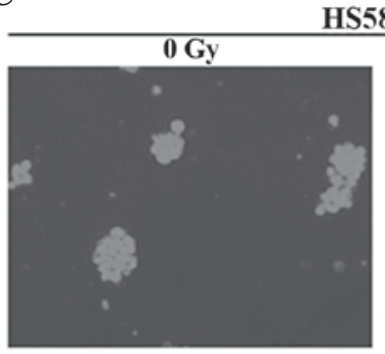

HS587-T

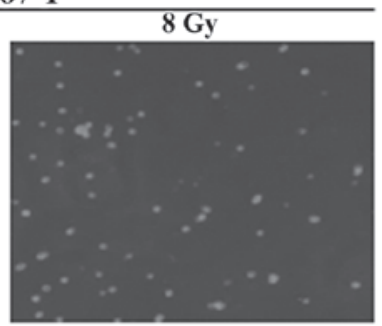

D

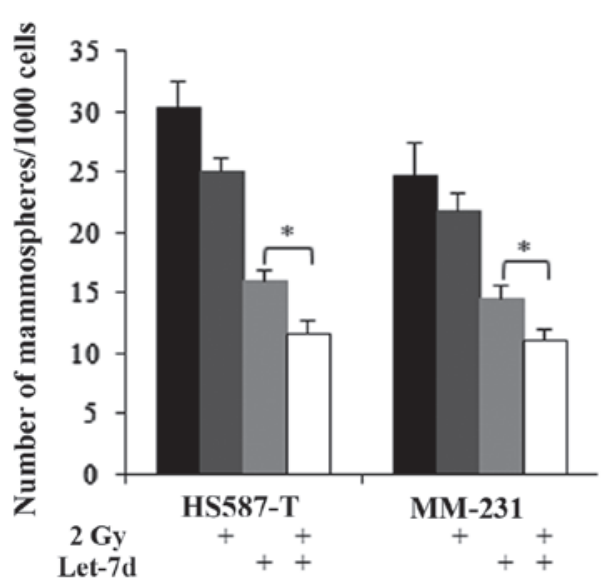

$\mathbf{E}$
B
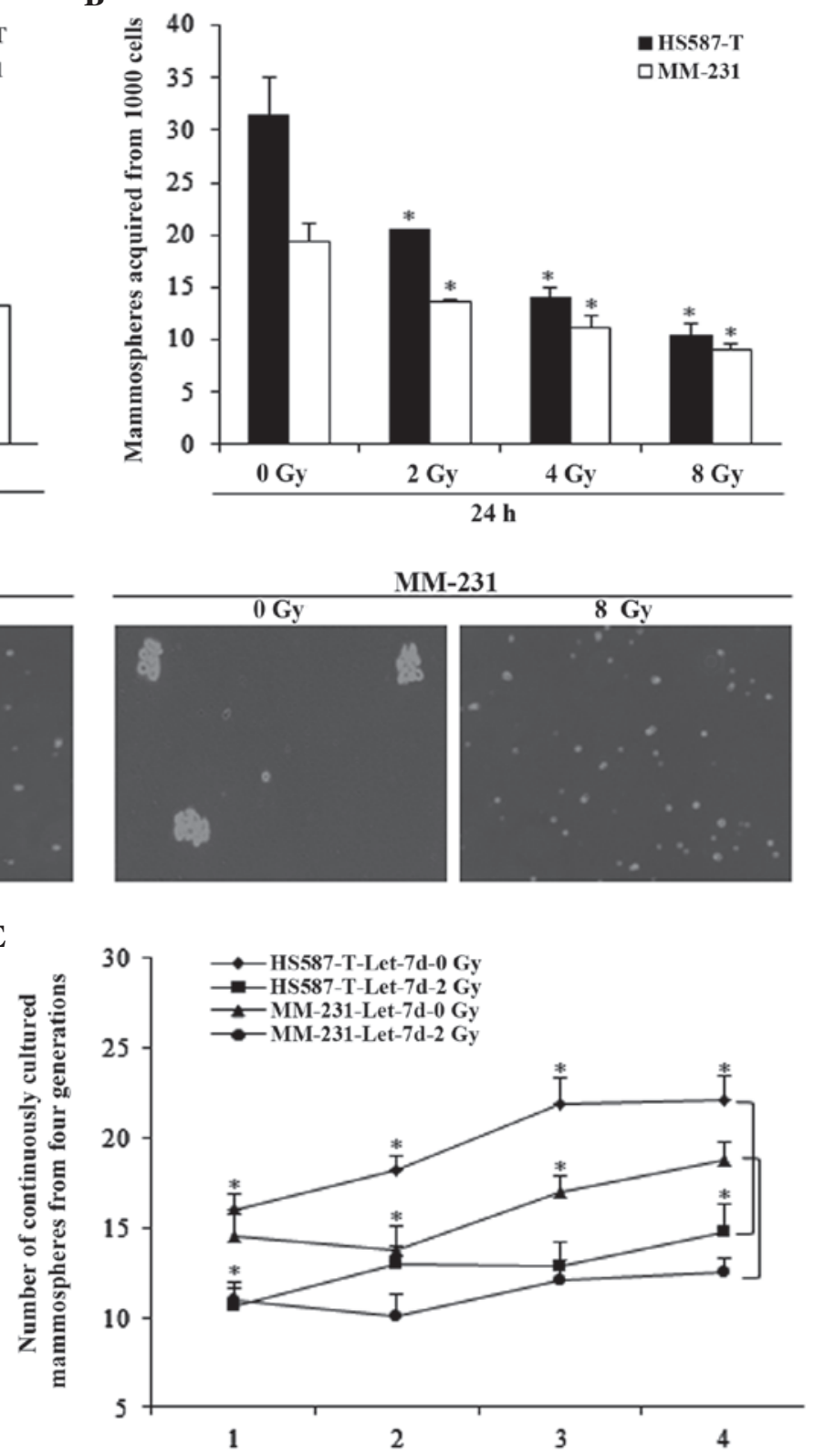

Figure 2. Let-7d sensitized triple negative breast cancer stem cells to radiation-induced self-renewal inhibition. (A) 4 Gy of radiation treatment for $12 \mathrm{~h}$ and (B) 2 Gy radiation treatment for $24 \mathrm{~h}$ induced significant inhibition of the self-renewal ability of HS587-T and MM-231 cells. The 2 Gy radiation dose for $12 \mathrm{~h}$ was then selected for further research. (C) Representative images of mammospheres acquired from 8 Gy radiation-treated HS587-T and MM-231 cells. (D) 2 Gy radiation exhibited a stronger inhibition of the self-renewal ability of HS587-T and MM-231 cells when in combination with let-7d. (E) The combination of let- $7 \mathrm{~d}$ and $2 \mathrm{~Gy}$ of radiation continuously inhibited the stem cell numbers in four generations of mammospheres. ${ }^{*} \mathrm{P}<0.01$ vs. the $0 \mathrm{~Gy}$ group.

was investigated in continuously cultured mammospheres, with the results indicating that the combination significantly inhibited the number of mammospheres, indicating the effects of 2 Gy of radiation (Fig. 2E).

Let-7d inhibited TNBC stem cells through the cyclin D1/Akt1/Wnt1 pathway. The cyclin D1/Akt1/Wnt1 pathway, which may contribute to let-7d and radiation-induced stem cell repression, was detected using western blotting, a luciferase assay and immunofluorescence. Let-7d and radiation were observed to suppress the self-renewal ability of HS587-T and MM-231 cells via the cyclin D1/Akt1/Wnt1 pathway, and let-7d significantly sensitized the effects of 2 Gy radiation
(Fig. 3A). Through using binding site predication software, no complementary sequences were recognized between let-7d and Akt1, TCF-4 and $\beta$-catenin (data not shown). Complementary binding sequences were only identified in the 3'UTR of cyclin D1 and let-7d (Fig. 3B). Let-7d was observed to reduce the luciferase activity of cyclin D1 containing the wildtype 3'UTR, with no functions on the mutant 3'UTR (Fig. 3B and C). However, let-7d was not identified to significantly inhibit TCF-4 and $\beta$-catenin (Fig. 3B and C). Immunostaining of TNBC cells demonstrated that cyclin D1 was reduced in the let-7d-overexpressed group, while no alterations in TCF-4 were observed, suggested that let-7d functioned via the cyclin D1/Akt1/Wnt1 pathway (Fig. 3D). 
A

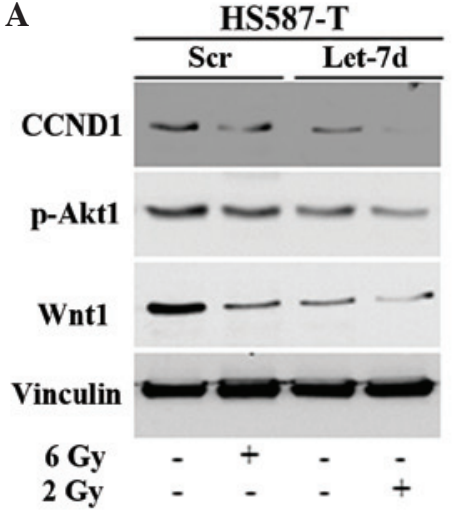

MM-231

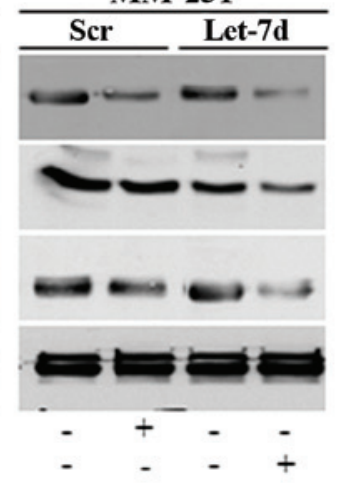

\begin{tabular}{l} 
Human CCND1 NM_053056 3'UTR length: 3192 \\
\hline 5' GUUGCUGUUUCACAA
\end{tabular}

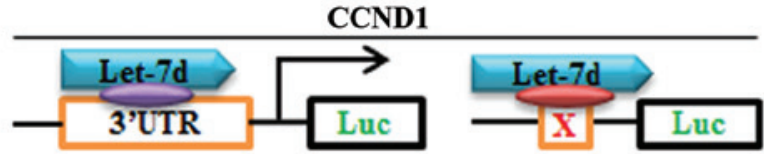

TCF-4, $\beta$-catenin

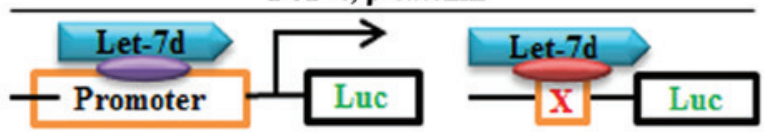

C
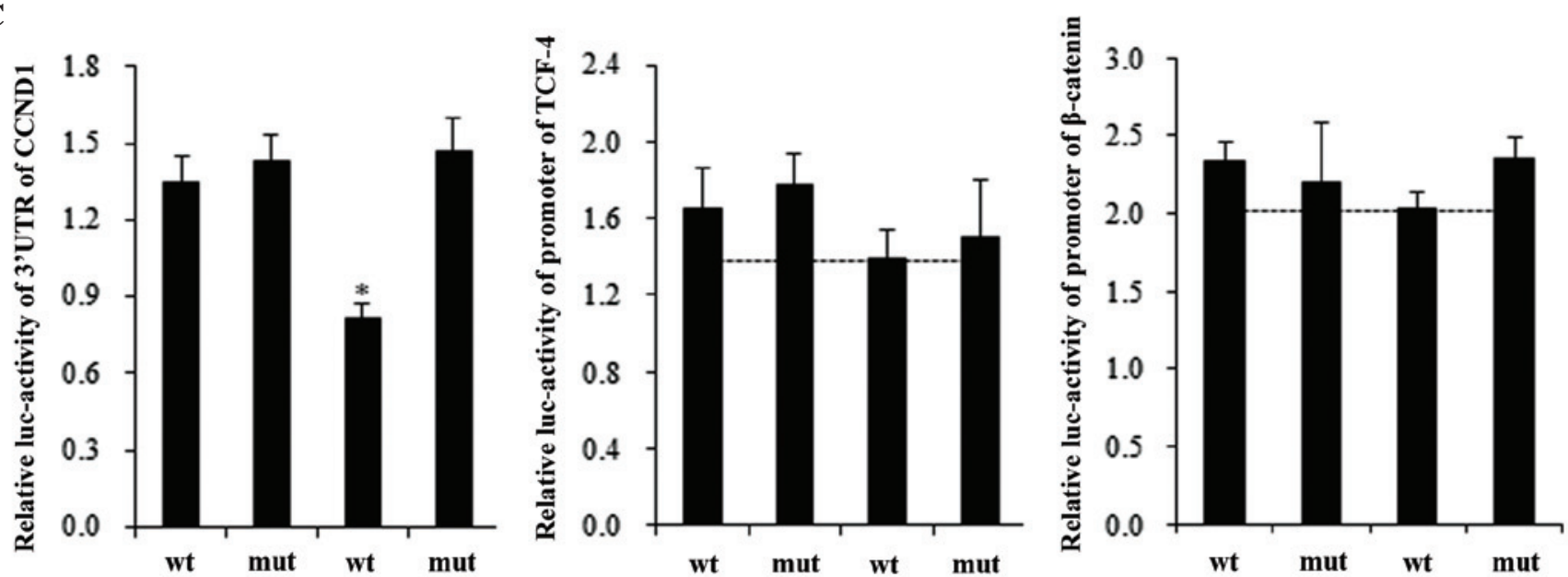

D

\section{DAPI}
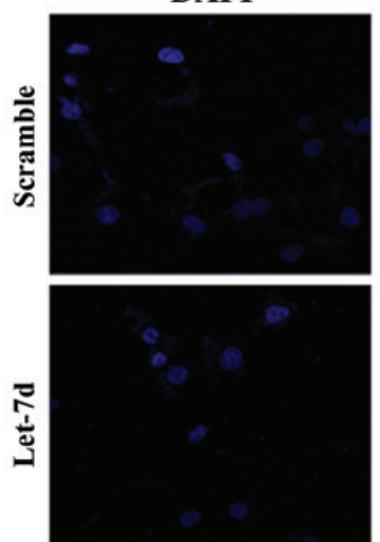

RFP
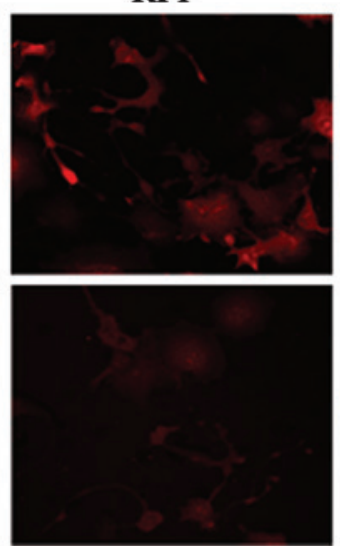
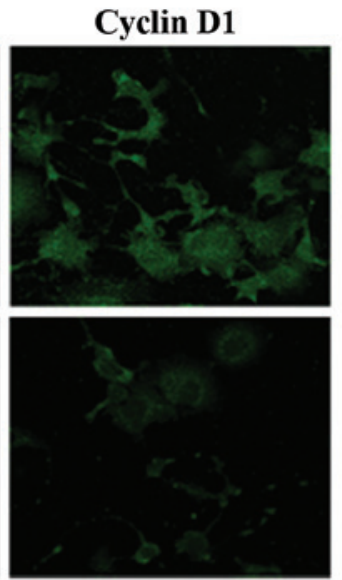
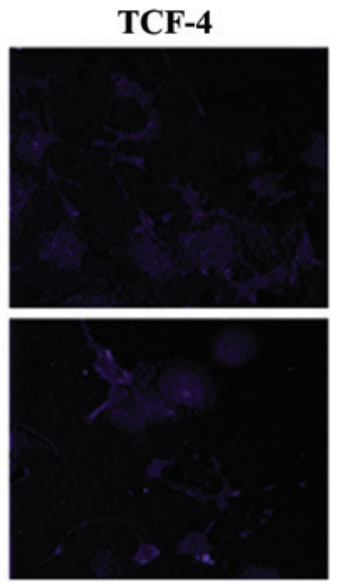
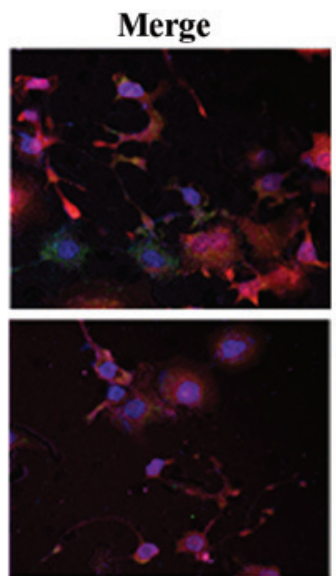

Figure 3. Let-7d inhibited the triple negative breast cancer stem cells through repressing the CCND1/Akt1/Wnt pathway. Western blotting, relative luciferase activity and immunofluorescence were measured in HS587-T and MM-231 cells. (A) Let-7d alone inhibited the CCND1/Akt1/Wnt pathway, and strengthened the 2 Gy radiation-induced suppression of the CCND1/Akt1/Wnt pathway. (B) No binding sites were identified between let-7d and the 3'UTR of Akt1, TCF-4 or $\beta$-catenin, and a common binding site was only identified between CCND1 and let-7 miRNAs, with the schematic diagram illustrating the mechanisms by which let-7d affects the CCND1/Akt1/Wnt pathway. (C) Marked reduction was observed following insertion of the 3'UTR of wt CCND1 into let-7d-overexpressed cells, however not with the insertion of mut CCND1. The reduction of TCF- 4 and the $\beta$-catenin promoter were not significant in let-7d-overexpressed HS587-T and MM-231 cells. "P<0.01 vs. the wt control group. (D) Immunostaining indicated a reduction in CCND1 in let-7d-overexpressed cells, while no alterations in TCF-4 were identified. Akt1, protein kinase B; Wnt, wingless type MMTV integration site family member 1; UTR, untranslated region; TCF-4, T-cell factor 4; wt, wildtype; mut, mutant; p-, phosphorylated; Luc, luciferase; DAPI, 4',6-diamidino-2-phenylindole; RFP, red fluorescent protein (from let-7 lentivirus); CCND1, cyclin D1.

Reduced Aktl phosphorylation and Wntl inhibition are required for let-7d sensitization of radiation through cyclin D1/Akt1/Wntl signaling. Let-7d inhibited Akt1/Wnt1 activity through inhibition of cyclin D1, and the small interfering RNA did not significantly induce cyclin D1 repression in let-7d-overexpressed HS587-T and MM-231 cells (Fig. 4A). The inhibition of cyclin D1 in let-7d-overexpressed cells did not significantly reduce the mammosphere number, 
A

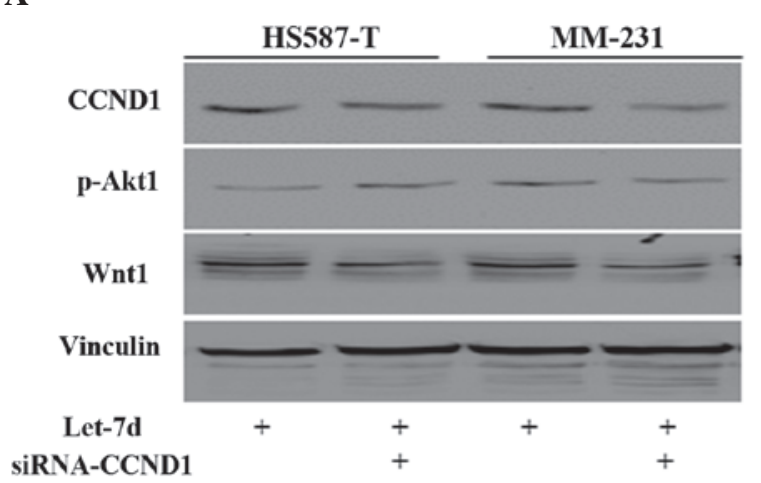

C

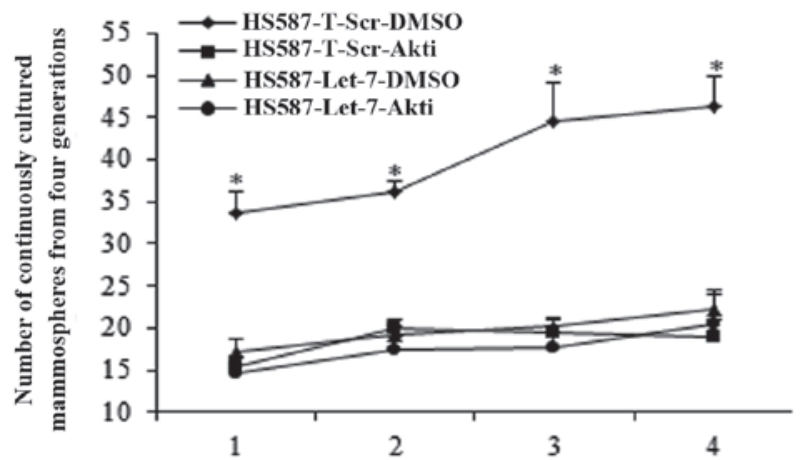

E

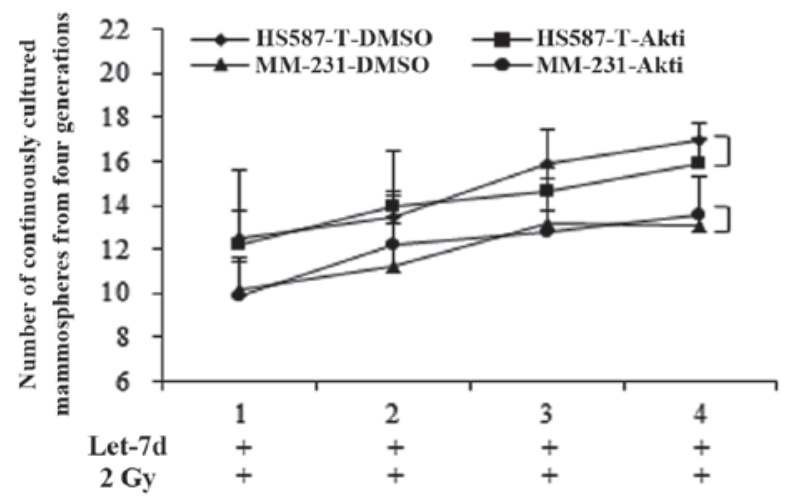

B

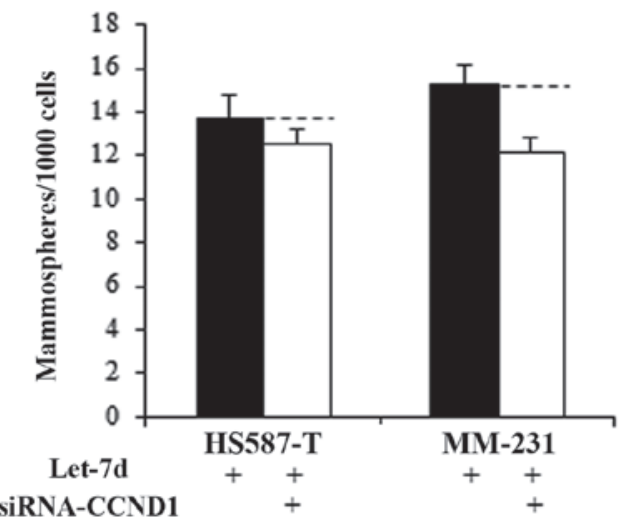

D

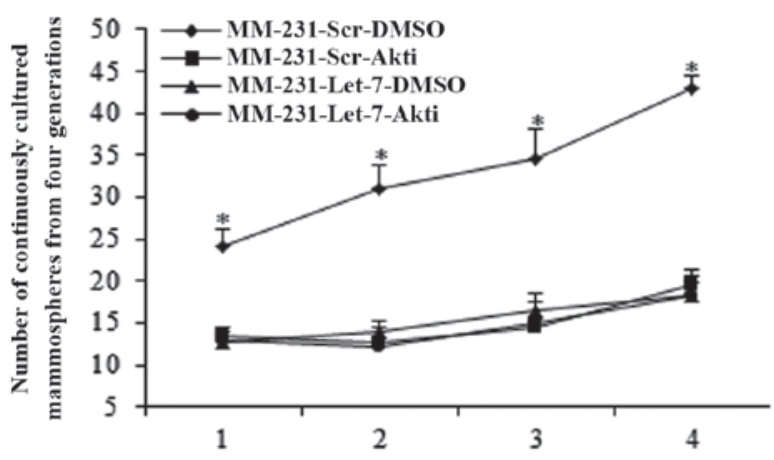

F

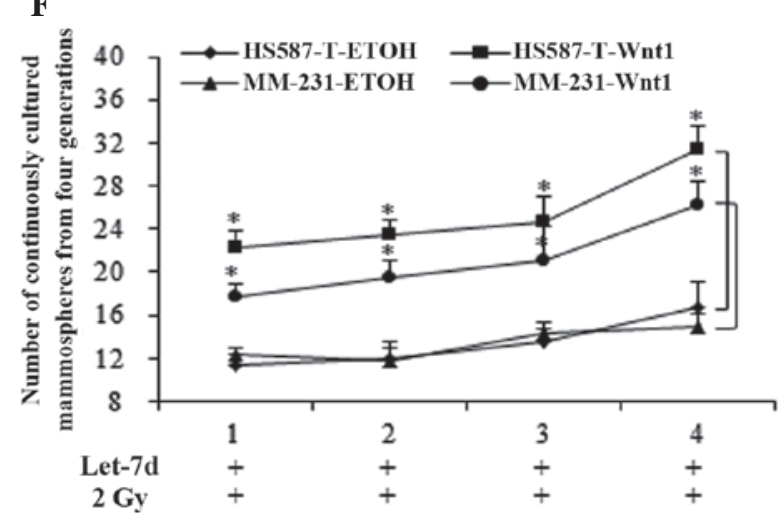

Figure 4. Let-7d sentisized breast cancer cells to the radiation response through inhibiting cyclin D1/Akt1/Wnt signaling. (A) Let-7d functioned through inhibition of cyclin D1, then inhibited the Akt phosphorylation and Wnt1 activation. (B) The inhibition of cyclin D1 in let-7d-overexpressed cells did not reduce the mammosphere number significantly, compared with the let-7d group, P>0.05. Akt inhibitors significantly inhibited the MFE of (C) HS587-T and (D) MM-231 cells of the scramble group, ${ }^{*} \mathrm{P}<0.01$ vs. the DMSO control group, with no significant alterations identified in cells infected with let-7d. (E and F) Akt phosphorylation inhibition resulting from Akt inhibitors did not influence the MFE of let-7d- and radiation-treated cells. (F) The restoration of Wnt1 abolished let-7d induction of radiation sensitization, ${ }^{*} \mathrm{P}<0.01$. Akt, protein kinase B; Wnt, wingless type MMTV integration site family member 1 ; MFE, mammosphere formation efficiency; siRNA, small interfering RNA; DMSO, dimethyl sulfoxide; Scr, scramble; ETOH, ethanol.

when compared with the let-7d group (Fig. 4B). Akt1 inhibitors and recombinant Wnt1 protein were used to identify the respective roles of Akt1 and Wnt1. Let-7d functioned through Akt inhibition, and the use of Akti-1/2 in the scramble group significantly inhibited the MFE of HS587-T (Fig. 4C) and MM-231 (Fig. 4D) cells ( $\mathrm{P}<0.01)$, with no significant alterations identified between let-7d and let-7d-Akti-1/2 (Fig. 4C and D). To elucidate whether inhibition of cyclin D1/Akt1/Wnt1 signaling accounts for let-7d-induced sensitization of the radiation response, Akti-1/2 and Wnt1 protein were used in cells treated with combination let-7d and 2 Gy radiation. Akt phosphorylation inhibition did not influence the MFE of let-7d and radiation-treated cells, indicating that let- $7 \mathrm{~d}$ functioned through Akt phosphorylation inhibition (Fig. 4E). The addition of Wnt1 significantly reversed let-7d-mediated induction of the radiation response $(\mathrm{P}<0.01$; Fig. $4 \mathrm{~F})$.

\section{Discussion}

Previous studies have indicated that the restoration of let-7 in tumors effectively inhibited cell proliferation and invasion, and sensitized the resistant cancer cells to chemotherapy $(22,23)$. Studies have previously investigated the function of let-7 on the self-renewal of CSCs, indicating 
that let-7 restoration may be utilized in therapy of breast cancer $(8,21,24)$. TNBC is more malignant and responds more poorly to chemotherapy and endocrine therapy than other subtypes of breast cancer, therefore it is important to investigate novel therapeutic options that may improve the prognosis of patients with TNBC. $\beta$-catenin controls cell adhesion and proliferation, stimulating TCF to markedly upregulate oncogenes (25), thus serves a major role in breast cancer, including TNBC $(26,27)$. Wnt1 stimulates the Wnt $1 / \beta$-catenin/TCF pathway and regulates the transcription of TCF motif activators, thus stimulating the self-renewal of CSCs (28-30).

To investigate the role of let-7 in breast cancer, let-7 miRNA signatures were investigated in multiple breast cancer cell lines. It was identified that let-7d was significantly reduced in triple negative HS587-T and MDA-MB-231 cells, indicating a potentially important role for let-7. Let-7d-overexpressing HS587-T and MDA-MB-231 were then constructed, and it was demonstrated that let-7d exhibited a strong inhibition on MFE, continuously inhibiting the self-renewal ability in up to four generations of cells. Radiation resulted in self-renewal inhibition. It was identified that 2 Gy radiation alone resulted in marginal inhibition on TNBC stem cells; however, when in combination with let-7d, 2 Gy radiation significantly suppressed the number of mammospheres. Through bioinformatics analysis and western blotting, the possible genes accounting for let-7-induced mammosphere inhibition were assessed. Let-7d was observed to inhibit cyclin D1 expression levels by directly inhibiting the cyclin D1 3'UTR, thus suppressing cyclin D1/Akt1/Wnt1 activity. The Akt inhibitor and Wnt1 activator were used to investigate let-7d-induced sensitization to radiotherapy. It was observed that let-7d functioned through cyclin D1/Akt1, with no significant alterations identified between let-7d and the Akt1 inhibitor, however reversal of Wnt1 inhibition abolished the functions of let-7d.

In conclusion, the results of the current study indicated that let-7d significantly inhibited the malignant behaviors of TNBC cells in vitro, and suppressed the self-renewal abilities of CSCs. Furthermore, in HS587-T and MM-231 cells, it was identified that let-7 functioned through inhibiting the cyclin D1/Akt1/Wnt1 pathway, and sensitized TNBC to radiation therapy-induced renewal repression (31). The repression of the Akt1/Wnt1 pathway in CSCs contributed to let-7-induced radiation sensitization, helping to inhibit the self-renewal of stem cells and eliminate the tumor group. The results of the current study aid in the understanding of the mechanisms through which let-7 regulated TNBC, and suggest that restoration of the let-7 family, particularly let-7d in TNBC, may be a novel therapeutic target.

\section{Acknowledgements}

The authors would like to thank the staff of the Central Laboratory of the First Affiliated Hospital of Zhengzhou University (Zhengzhou, China), for their technical assistance.

\section{References}

1. Jemal A, Bray F, Center MM, Ferlay J, Ward E and Forman D: Global cancer statistics. CA Cancer J Clin 61: 69-90, 2011.
2. Desmedt C, Haibe-Kains B, Wirapati P, Buyse M, Larsimont D, Bontempi G, Delorenzi M, Piccart M and Sotiriou C: Biological processes associated with breast cancer clinical outcome depend on the molecular subtypes. Clin Cancer Res 14: 5158-5165, 2008.

3. Tran B and Bedard PL: Luminal-B breast cancer and novel therapeutic targets. Breast Cancer Res 13: 221, 2011.

4. Hayes EL and Lewis-Wambi JS: Mechanisms of endocrine resistance in breast cancer: An overview of the proposed roles of noncoding RNA. Breast Cancer Res 17: 40, 2015.

5. Sotiriou C, Neo SY, McShane LM, Korn EL, Long PM, Jazaeri A, Martiat P, Fox SB, Harris AL and Liu ET: Breast cancer classification and prognosis based on gene expression profiles from a population-based study. Proc Natl Acad Sci USA 100: 10393-10398, 2003.

6. Assi HA, Khoury KE, Dbouk H, Khalil LE, Mouhieddine TH and El Saghir NS: Epidemiology and prognosis of breast cancer in young women. J Thorac Dis 5 (Suppl 1): S2-S8, 2013.

7. Sun X, Jiao X, Pestell TG, Fan C, Qin S, Mirabelli E, Ren H and Pestell RG: MicroRNAs and cancer stem cells: The sword and the shield. Oncogene 33: 4967-4977, 2014.

8. Rothschild SI: microRNA therapies in cancer. Mol Cell Ther 2: 7, 2014.

9. Costa PM and Pedroso de Lima MC: MicroRNAs as molecular targets for cancer therapy: On the modulation of microRNA expression. Pharmaceuticals (Basel) 6: 1195-1220, 2013.

10. Sun X, Tang SC, Xu C, Wang C, Qin S, Du N, Liu J, Zhang Y, Li X, Luo G, et al: Dicer regulated let-7 expression levels in p53-induced cancer repression requires cyclin D1. J Cell Mol Med 19: 1357-1365, 2015.

11. Cai J, Guan H, Fang L, Yang Y, Zhu X, Yuan J, Wu J and Li M: MicroRNA-374a activates Wnt/ $\beta$-catenin signaling to promote breast cancer metastasis. J Clin Invest 123: 566-579, 2013.

12. Laezza C, d'Alessandro A, Malfitano AM and Bifulco M: Anandamide inhibits the $\mathrm{Wnt} / \beta$-catenin signalling pathway in human breast cancer MDA MB 231 cells. Eur J Cancer 49: 2066-2067, 2013.

13. Malanchi I, Santamaria-Martinez A, Susanto E, Peng H, Lehr HA, Delaloye JF and Huelsken J: Interactions between cancer stem cells and their niche govern metastatic colonization. Nature 481: 85-89, 2011.

14. Livak KJ and Schmittgen TD: Analysis of relative gene expression data using real-time quantitative PCR and the 2(-Delta Delta C(T)) method. Methods 25: 402-408, 2001.

15. Cicalese A, Bonizzi G, Pasi CE, Faretta M, Ronzoni S, Giulini B, Brisken C, Minucci S, Di Fiore PP and Pelicci PG: The tumor suppressor p53 regulates polarity of self-renewing divisions in mammary stem cells. Cell 138: 1083-1095, 2009.

16. Oliveras-Ferraros C,CufiS, Vazquez-Martin A, Torres-Garcia VZ, Del Barco S, Martin-Castillo B and Menendez JA: Micro (mi) RNA expression profile of breast cancer epithelial cells treated with the anti-diabetic drug metformin: Induction of the tumor suppressor miRNA let-7a and suppression of the TGF $\beta$-induced oncomiR miRNA-181a. Cell Cycle 10: 1144-1151, 2011.

17. Sun Y, Wang Y, Fan C, Gao P, Wang X, Wei G and Wei J: Estrogen promotes stemness and invasiveness of ER-positive breast cancer cells through Gli1 activation. Mol Cancer 13: 137, 2014.

18. Zhou J, Wang C, Wang Z, Dampier W, Wu K, Casimiro MC, Chepelev I, Popov VM, Quong A, Tozeren A, et al: Attenuation of Forkhead signaling by the retinal determination factor DACH1. Proc Natl Acad Sci USA 107: 6864-6869, 2010.

19. Johnson CD, Esquela-Kerscher A, Stefani G, Byrom M, Kelnar K, Ovcharenko D, Wilson M, Wang X, Shelton J, Shingara J, et al: The let-7 microRNA represses cell proliferation pathways in human cells. Cancer Res 67: 7713-7722, 2007.

20. Tsang WP and Kwok TT: Let-7a microRNA suppresses therapeutics-induced cancer cell death by targeting caspase- 3 . Apoptosis 13: 1215-1222, 2008.

21. Saridaki Z, Weidhaas JB, Lenz HJ, Laurent-Puig P, Jacobs B, De Schutter J, De Roock W, Salzman DW, Zhang W, Yang D, et al: A let-7 microRNA-binding site polymorphism in KRAS predicts improved outcome in patients with metastatic colorectal cancer treated with salvage cetuximab/panitumumab monotherapy. Clin Cancer Res 20: 4499-4510, 2014.

22. Worringer KA, Rand TA, Hayashi Y, Sami S, Takahashi K, Tanabe K, Narita M, Srivastava D and Yamanaka S: The let-7/LIN-41 pathway regulates reprogramming to human induced pluripotent stem cells by controlling expression of prodifferentiation genes. Cell Stem Cell 14: 40-52, 2014.

23. Anastas JN and Moon RT: WNT signalling pathways as therapeutic targets in cancer. Nat Rev Cancer 13: 11-26, 2013. 
24. Ohno S, Takanashi M, Sudo K, Ueda S, Ishikawa A, Matsuyama N, Fujita K, Mizutani T, Ohgi T, Ochiya T, et al: Systemically injected exosomes targeted to EGFR deliver antitumor microrna to breast cancer cells. Mol Ther 21: 185-191, 2013.

25. Laezza C, D'Alessandro A, Paladino S, Maria Malfitano A, Chiara Proto M, Gazzerro P, Pisanti S, Santoro A, Ciaglia E and Bifulco M; Endocannabinoid Research Group: Anandamide inhibits the Wnt $/ \beta$-catenin signalling pathway in human breast cancer MDA MB 231 cells. Eur J Cancer 48: 3112-3122, 2012.

26. Taurin S, Sandbo N, Yau DM, Sethakorn N and Dulin NO Phosphorylation of beta-catenin by PKA promotes ATP-induced proliferation of vascular smooth muscle cells. Am J Physiol Cell Physiol 294: C1169-C1174, 2008.
27. Midwood KS and Orend G: The role of tenascin-C in tissue injury and tumorigenesis. J Cell Commun Signal 3: 287-310, 2009.

28. Choi AR, Park JR, Kim RJ, Kim SR, Cho SD, Jung JY and Nam JS: Inhibition of Wnt1 expression reduces the enrichment of cancer stem cells in a mouse model of breast cancer. Biochem Biophys Res Commun 425: 436-442, 2012.

29. Katoh M and Katoh M: WNT signaling pathway and stem cell signaling network. Clin Cancer Res 13: 4042-4045, 2007.

30. He B and Jablons DM: Wnt signaling in stem cells and lung cancer. In: Cancer Stem Cells. Wiestler OD, Haendler B and Mumberg D (eds). Springer-Verlag, Berlin, pp27-58, 2007.

31. Sun X, Qin S, Fan C, Xu C, Du N and Ren H: Let-7: A regulator of the ERalpha signaling pathway in human breast tumors and breast cancer stem cells. Oncol Rep 29: 2079-2087, 2013. 